\title{
Affective network and default mode network in depressive adolescents with disruptive behaviors
}

This article was published in the following Dove Press journal:

Neuropsychiatric Disease and Treatment

31 December 2015

Number of times this article has been viewed

\author{
Sun Mi Kim' \\ Sung Yong Park' \\ Young In Kim' \\ Young Don Son ${ }^{2}$ \\ Un-Sun Chung 3,4 \\ Kyung Joon Min' \\ Doug Hyun Han'
}

'Department of Psychiatry, School of Medicine, Chung-Ang University, Seoul, ${ }^{2}$ Department of Biomedical

Engineering, Gachon University of Medicine and Science, Incheon,

${ }^{3}$ Department of Psychiatry,

School of Medicine, Kyungpook

National University, ${ }^{4}$ School Mental

Health Resources and Research

Center, Kyungpook National

University Children's Hospital,

Daegu, South Korea
Correspondence: Doug Hyun Han Department of Psychiatry, School of Medicine, Chung-Ang University, Heukseok-ro 102, Dongjack-gu,

Seoul 156-755, South Korea

Tel +82 262993132

Email hduk@yahoo.com
Aim: Disruptive behaviors are thought to affect the progress of major depressive disorder (MDD) in adolescents. In resting-state functional connectivity (RSFC) studies of MDD, the affective network (limbic network) and the default mode network (DMN) have garnered a great deal of interest. We aimed to investigate RSFC in a sample of treatment-naïve adolescents with MDD and disruptive behaviors.

Methods: Twenty-two adolescents with MDD and disruptive behaviors ( ${ }_{\text {disrup }}$ MDD) and 20 age- and sex-matched healthy control (HC) participants underwent resting-state functional magnetic resonance imaging (fMRI). We used a seed-based correlation approach concerning two brain circuits including the affective network and the DMN, with two seed regions including the bilateral amygdala for the limbic network and the bilateral posterior cingulate cortex (PCC) for the DMN. We also observed a correlation between RSFC and severity of depressive symptoms and disruptive behaviors.

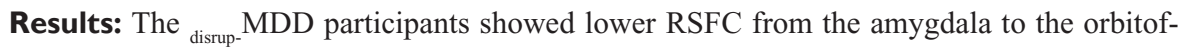
rontal cortex and parahippocampal gyrus compared to HC participants. Depression scores in disrup-MDD participants were negatively correlated with RSFC from the amygdala to the right

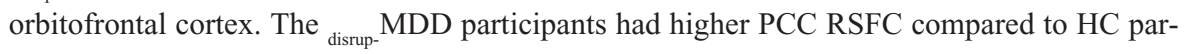
ticipants in a cluster that included the left precentral gyrus, left insula, and left parietal lobe. Disruptive behavior scores in disrup- MDD patients were positively correlated with RSFC from the PCC to the left insular cortex.

Conclusion: Depressive mood might be correlated with the affective network, and disruptive behavior might be correlated with the DMN in adolescent depression.

Keywords: resting-state functional connectivity, major depressive disorder, disruptive behaviors, limbic network, default mode network

\section{Introduction}

\section{Major depressive disorder and disruptive behavior in adolescence}

Major depressive disorder (MDD) is a common psychiatric disorder in adolescence, with a prevalence rate higher than $4 \% .{ }^{1}$ Compared to classical depressive features, including low self-esteem, excessive guilt, suicidal behaviors or thoughts, sleep and appetite disturbance, and agitation in adults, adolescent depression tends to be more complex, presenting with comorbidities (anxiety disorder, alcohol and substance use, conduct disorder, or oppositional defiant disorder [ODD]). ${ }^{2}$ Among various comorbidities, ODD might be one of the strongest comorbid conditions that affects the progress of adolescent depressive symptoms. ${ }^{2}$ Moreover, it has been suggested that the irritable sub-component of ODD shares a genetic association with major depression and increases the risk of depression. ${ }^{3}$ The clinical symptoms of ODD or conduct disorder have also been frequently observed in adolescents with attention-deficit hyperactivity 
disorder (ADHD). Therefore, many clinicians are interested in differentiating MDD from ADHD when they observe disruptive behaviors and irritability in adolescents. ${ }^{4}$

It has been suggested that adolescents with MDD and disruptive behavior differ from those with MDD and no disruptive behavior symptoms. Children with MDD comorbid with conduct disorders were more frequently comorbid with substance use disorder and showed greater irritability and worse outcomes. ${ }^{5}$ The difference in severity of emotional symptoms related to depression between adolescents with MDD comorbid with and without conduct disorder remain controversial. Ezpeleta et $\mathrm{al}^{5}$ reported the accentuation of emotional symptoms and functional impairment in the comorbid condition. In contrast, Simic and Fombonne ${ }^{6}$ reported fewer emotional symptoms in the comorbid condition.

\section{Resting-state functional connectivity for depression and disruptive behaviors}

Neuroimaging techniques representing brain circuit-human behavior relationships can provide potential biological markers of mental disorders. ${ }^{7}$ Of existing neuroimaging techniques, resting-state functional connectivity (RSFC) indicates information about intrinsic connectivity within brain networks without the need for specific challenges or task stimulations. ${ }^{8}$ Moreover, because it enables a focus on brain regions of interest (ROI), including the amygdala, striatum, prefrontal cortex, and insula, which have been demonstrated to be candidate regions in previous studies with other modalities, ${ }^{9,10}$ RSFC studies provide an understanding of the pathophysiologic brain processing associated with behaviorally and emotionally labile adolescents. ${ }^{11}$

In RSFC studies of MDD, the affective network (limbic network) and the default mode network (DMN) have garnered a great deal of interest. The affective network includes the amygdala, orbitofrontal cortex (OFC), temporal cortex, pallidum, and insular cortex. ${ }^{12}$ The role of the affective network is emotion regulation and processing. ${ }^{12}$ In resting-state functional magnetic resonance imaging (fMRI) analysis of the amygdala seed, several studies have reported abnormalities within the affective network in patients with MDD. ${ }^{11,13,14}$ Luking et $\mathrm{al}^{13}$ have reported that RSFC from the amygdala to prefrontal regions and from the amygdala to the striatum in adolescents with MDD is decreased compared to that in healthy control (HC) participants. Pannekoek et al ${ }^{14}$ have reported that RSFC from the right amygdala to the left anterior cingulate cortex in adolescents with MDD is decreased compared to that in $\mathrm{HC}$ participants. Still, few studies on RSFC in the affective network have been conducted in patients with conduct or ODD. The majority of functional neuroimaging studies in conduct disorder patients have involved eventrelated fMRI using emotion processing tasks. ${ }^{15}$ Bebko et a ${ }^{11}$ have found that RSFC from the amygdala to the insula is negatively correlated with depressive symptoms and behavioral dysregulation symptom scores in adolescents with behavioral and emotional dysregulation.

The DMN includes the posterior cingulate cortex (PCC), precuneus, medial frontal cortex, ventral anterior cingulate cortex, and lateral and inferior parietal cortices. ${ }^{8}$ Interestingly, because the DMN is activated at rest and not during task stimulation, it is posited to be related to self-referential processing. ${ }^{16}$ Among components of the DMN, the PCC was the most consistently selected region in resting-state fMRI. The PCC is thought to be associated with the monitoring of internal and external environments. ${ }^{8}$ In a review of the DMN and patients with MDD, Nejad et a ${ }^{17}$ suggest that self-referential processing in patients with MDD is associated with increased RSFC in the DMN. In a study conducted in adolescents who had comorbid conduct disorder and substance use disorder, ${ }^{18}$ reduced activity was shown in several DMN regions thought to play a role in introspective processing and future thought.

\section{The aim of this study}

Based on previous studies, we sought to investigate RSFC in a sample of treatment-naïve adolescents with MDD and disruptive behaviors. We used a seed-based correlation approach concerning two brain circuits including the affective network and the DMN. In addition, we observed the correlation between RSFC and severity of depressive symptoms and disruptive behaviors.

\section{Methods}

\section{Participants}

A total of 35 adolescents with MDD and disruptive behaviors (disrup- MDD) who visited the Department of Psychiatry at Chung-Ang University Hospital were screened. All patients were experiencing their first episode of depression and had no history of psychiatric treatment or medication. Inclu-

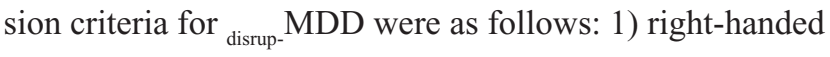
adolescents aged $13-18$ years; 2 ) demonstrating at least one disruptive behavior (consistent defiance of authoritative figures, inability to take responsibility for bad behavior, regular temper tantrums, vengeful behavior and resentment, aggressiveness toward people or animals, destroying property, stealing and lying, bullying, and constant rule breaking), in addition to meeting diagnostic criteria for MDD 
according to the Diagnostic and Statistical Manual of Mental Disorders, text revision (DSM-IV-TR); and 3) a full-scale intelligence score $(\mathrm{IQ})>80$. Exclusion criteria for disrup- $_{-}$MDD were as follows: 1) primary DSM-IV-TR clinical diagnosis of another disorder, including ADHD, bipolar disorder, Tourette's syndrome, developmental disorder, anxiety disorder, or psychotic disorder; 2) general magnetic resonance imaging (MRI) contraindications including claustrophobia and previous metal insertion; and 3) substance abuse. HC participants had similar inclusion and exclusion criteria except for MDD and disruptive behavior conditions. This study was approved by the Chung-Ang University Hospital Institutional Review Board. Written, informed consent and assent were provided by adolescents and their parents.

\section{Measures}

Both patients and $\mathrm{HC}$ participants were screened using the Kiddie-Schedule for Affective Disorders and Schizophrenia-Present and Lifetime Version-Korean version (K-SADS-PL-K). ${ }^{19}$ Depressive symptoms, disruptive behaviors, clinical symptoms of ADHD, and anxiety were assessed with the Children's Depression Inventory (CDI), ${ }^{20}$ the Rating Scale for Disruptive Behavior Disorder (DBDS), ${ }^{21}$ the Korean ADHD Rating Scale (K-ARS), ${ }^{22}$ and the Beck Anxiety Inventory, ${ }^{23}$ respectively. Additionally, all adolescents were tested with the Korean version of the Wechsler Intelligence Scales for Children. ${ }^{24}$ After screening and IQ assessment, a licensed child and adolescent psychiatrist confirmed diagnosis. Of the 35 patients screened, 13 adolescents were ruled out due to a diagnosis of $\operatorname{ADHD}(n=6)$, bipolar disorder $(n=3)$, anxiety disorder $(n=2)$, low IQ $(n=1)$, or autism spectrum disorder $(n=1)$. A final total of 22 adolescents with MDD and disruptive behaviors and 20 age- and sex-matched $\mathrm{HC}$ participants were included in data analyses.

\section{Procedure}

\section{Brain imaging data analysis}

We collected resting-state functional MRI (fMRI) data using a 3.0 Tesla TX MRI scanner (Philips, Eindhoven, the Netherlands). All images (230 volumes per participant) over 720 seconds were acquired with the following parameters: axially with an echo-planar imaging sequence, $\mathrm{TR} / \mathrm{TE}=3,000 / 40$ $\mathrm{ms}, 40$ slices, $64 \times 64$ matrix, $90^{\circ}$ flip angle, $230 \mathrm{~mm}$ field of view, and $3 \mathrm{~mm}$ section thickness without a gap. Data processing was carried out using the Data Processing Assistant for Resting-State fMRI (DPARSFA, http://www.restfmri.net) in Statistical Parameter Mapping (SPM8, http://www.fil.ion.ucl. ac.uk/spm/software/spm8/) and the Resting-State fMRI Data
Analysis toolkit (REST, http://resting-fmri.sourceforge.net). The first ten volumes from each run were discarded due to signal equilibration. As the preprocessing steps, images were corrected for slice acquisition delay between slices and were realigned to the first image in order to correct head movement. All images were spatially normalized to the standard Montreal Neurological Institute (MNI) space, spatially smoothed with a $4 \mathrm{~mm}$ FWHM 3D Gaussian kernel to reduce spatial noise, and linearly de-trended and temporally filtered with a bandwidth of $0.01-0.08 \mathrm{~Hz}$ to reduce the effects of low-frequency drift and high-frequency noise, respectively. Finally, the first eigenvector of the time series in the white matter mask and the first eigenvector of the times series in the cerebrospinal fluid mask were removed using linear regression in order to reduce physiological and other sources of noise and head motion-related covariates.

In both participant groups, RSFC was calculated from blood-oxygenation level-dependent signal using a seed-based approach. The mean time series of an ROI was extracted by Wake Forest University PickAtlas software, version 2.4 (http://fmri.wfubmc.edu/software/PickAtlas; Wake Forest University School of Medicine Location, Winston-Salem, NC, USA), ${ }^{25,26}$ and ROIs were predefined by automated anatomical labeling equipped with PickAtlas. Based on previous studies, ${ }^{7,11}$ bilateral amygdala and posterior cingulate cortices were selected as seed ROIs representing the affective limbic network and DMN, respectively. Two functional connectivity maps were generated by calculating Pearson's correlation coefficients of the average time-course signal of each seed ROI to every other voxel. The resultant connectivity maps were converted to normally distributed $z$-scores using Fisher's z-transform.

\section{Statistical analysis}

Demographic and clinical characteristics were analyzed using chi-square tests and Mann-Whitney $U$-tests with significance set at $P<0.05$. Based on previous studies, ${ }^{7,11}$ two seed regions including the bilateral amygdala (for the affective network) and the bilateral PCC (for the DMN) were selected. In the firstlevel analysis of the comparisons between disrup- $\mathrm{MDD}$ and control groups, seed-based FC analyses were performed using a two-sample $t$-test. In the second analysis, correlations between CDI scales (DBDS scales) and RSFC from the amygdala to the OFC and from the PCC to the insula were analyzed using regression. For multiple comparisons with $>40$ contiguous voxels in the cluster analysis, the resulting maps were set to a threshold of $P<0.05$ for family-wise corrected type I error rate using Monte Carlo simulation (derived from an uncorrected $P<0.001$ and 40 extended voxels). 


\section{Results}

\section{Demographic characteristics}

There were no significant differences in sex, age, years of education, IQ, or Beck Anxiety Inventory score between disrup- $\mathrm{MDD}$ and HC (Table 1). However, the mean CDI score (MDD: mean \pm standard deviation, $39.5 \pm 14.7$ vs HC: 15.3 \pm 8.1 ), DBDS score (MDD: $22.9 \pm 11.5$ vs HC: $5.1 \pm 3.6$ ), and K-ARS score (MDD: $11.3 \pm 4.0$ vs HC: $7.5 \pm 3.8$ ) in the disrup $M$ MD group were higher than those in the controls. There was significant correlation between DBDS score and CDI score $(r=0.44, P=0.04)$ and $\mathrm{K}$-ARS score $(r=0.49, P=0.02)$ in the ${ }_{\text {disrup }}$ MDD group. However, there was no significant correlation between DBDS score and other clinical measurements in the disrup- $_{\text {MDD group. }}$

\section{RSFC analysis}

\section{Affective network}

With respect to between-group analyses for the limbic net-

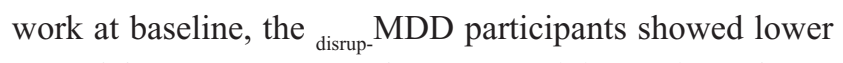
amygdala RSFC compared to HC participants in a cluster that included the right subcallosal and right parahippocampal gyrus (Figure 1; Table 2). CDI score in disrup- MDD patients was negatively correlated with RSFC from the amygdala to the right OFC (101 voxels; Pearson $r=-0.63, P<0.001$ ). There was no significant association between CDI score in $_{\text {disrup- }}$ MDD patients and RSFC from the PCC to other brain regions.

\section{Default mode network}

With respect to between-group analyses for the DMN at baseline, the disrup-MDD participants showed higher PCC RSFC compared to HC participants in a cluster that included the left precentral gyrus (white matter), left insula (Brodmann's area [BA] 13), and left parietal lobe (white matter)

Table I Demographic characteristics of the study participants

\begin{tabular}{llll}
\hline Characteristics & disrup- $M D D(\mathbf{n}=\mathbf{2 2})$ & $\mathbf{H C}(\mathbf{n}=\mathbf{2 0})$ & Statistics \\
\hline Sex (male/female) & $14 / 8$ & $14 / 6$ & $\chi^{2}=0.19, P=0.66$ \\
Age (years) & $13.9 \pm 1.6^{*}$ & $14.5 \pm 1.7$ & $z=1.16, P=0.22$ \\
Years of education & $6.0 \pm 1.6$ & $6.6 \pm 1.7$ & $z=1.16, P=0.21$ \\
IQ & $96.2 \pm 9.6$ & $100.8 \pm 9.4$ & $z=1.50, P=0.13$ \\
CDI & $40.0 \pm 7.3$ & $10.0 \pm 5.1$ & $z=5.46, P<0.01$ \\
DBDS & $22.9 \pm 11.4$ & $5.1 \pm 3.6$ & $z=4.98, P<0.01$ \\
K-ARS & $11.3 \pm 4.0$ & $7.5 \pm 3.8$ & $z=3.15, P<0.01$ \\
BAI & $8.0 \pm 4.1$ & $4.3 \pm 3.1$ & $z=1.01, P=0.30$ \\
\hline
\end{tabular}

Notes: *Mean \pm standard deviation. $P<0.05$, statistically significant.

Abbreviations: disrup. MDD, adolescents with major depressive disorder and disruptive behaviors; HC, healthy controls; IQ, intelligence quotient; CDI, Children's Depression Inventory; DBDS, Rating Scale for Disruptive Behavior Disorder; K-ARS, Korean ADHD Rating Scale; BAI, Beck Anxiety Inventory.
(Figure 2; Table 3). DBDS score in disrup $\mathrm{MDD}$ patients was positively correlated with RSFC from the PCC to the left insular cortex (91 voxels; Pearson $r=0.71, P<0.001$ ). There was no significant association between DBDS score in disrup. MDD patients and RSFC from the PCC to other brain regions.

\section{Discussion}

The current study found that disrup- MDD participants show different RSFC in the affective network and DMN compared to HC participants. The disrup MDD participants showed lower RSFC from the amygdala to the OFC and parahippocampal

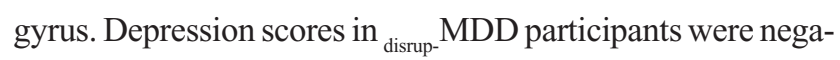
tively correlated with RSFC from the amygdala to the right

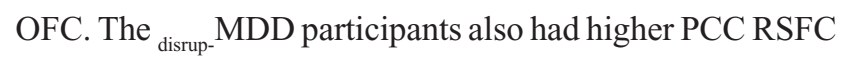
compared to $\mathrm{HC}$ participants in a cluster that included the left precentral gyrus, left insula, and left parietal lobe. Disruptive

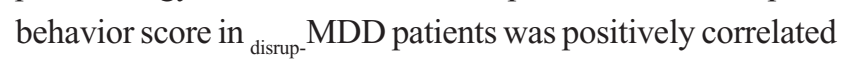
with RSFC from the PCC to the left insular cortex.

\section{RSFC from the amygdala to the OFC and parahippocampal gyrus}

Our current results are consistent with other RSFC studies showing lower RSFC in the affective network from the amygdala to the OFC and parahippocampal gyrus in adolescents with MDD. ${ }^{27-29}$ Lower RSFC between the amygdala and the OFC has also been reported in previous imaging studies, suggesting an inhibitory effect of the OFC..$^{27,28}$ As part of the prefrontal cortex, the OFC is known to regulate negative emotion. ${ }^{30}$ Disconnect between the amygdala and OFC might lead to a disruption of the OFC's ability to regulate emotion, especially negative emotions. ${ }^{27,28,30} \mathrm{Cul}-$ len et $\mathrm{al}^{29}$ reported that adolescents with MDD show lower RSFC from the amygdala to the hippocampus, parahippocampus, and brain stem. This RSFC is negatively correlated with depression and dysphoric score. The amygdala is thought to be richly connected with the parahippocampal gyrus. ${ }^{31,32}$ Enhanced connectivity between the amygdala and hippocampus and parahippocampal gyrus might facilitate memory modulation. ${ }^{33}$ The disconnect between the amygdala and parahippocampal gyrus might also be associated with depression, dysphoria, and lassitude. ${ }^{29,33}$ In contrast to RSFC, patients with MDD have also shown increased connectivity from the amygdala to the OFC in event-related fMRI studies involving memory of sad stimuli compared to that in $\mathrm{HCs} .{ }^{34}$ Taken together, these result indicate that the affective network in patients with MDD is underconnected during rest. However, compensatory processes to offset the hyperactivity 
A

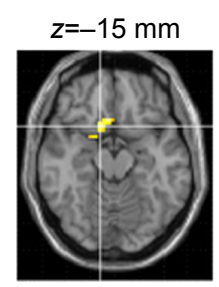

B

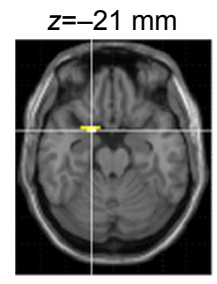

$z=-12 \mathrm{~mm}$

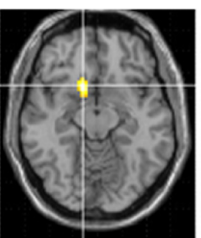

$z=-18 \mathrm{~mm}$

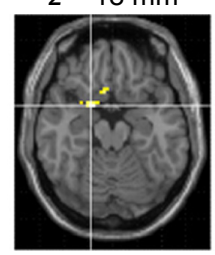

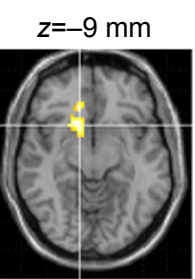

$z=-15 \mathrm{~mm}$

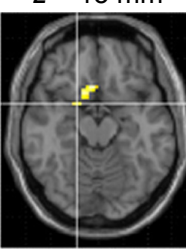

E

$E$
$\frac{0}{2}$
$\frac{0}{0}$
$\frac{\pi}{0}$
$m$

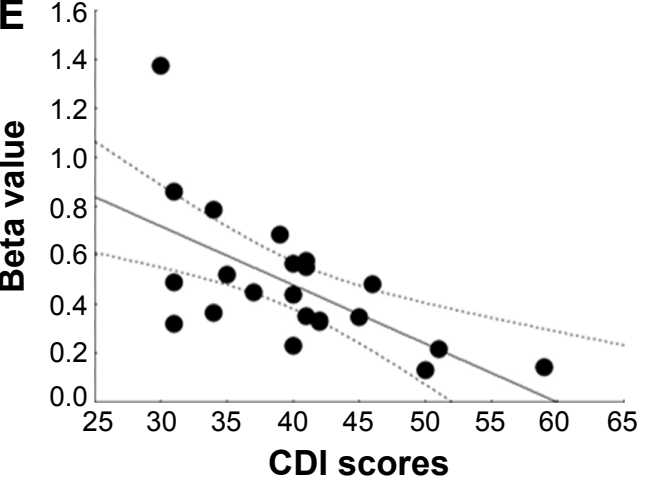

C

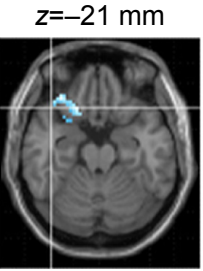

D
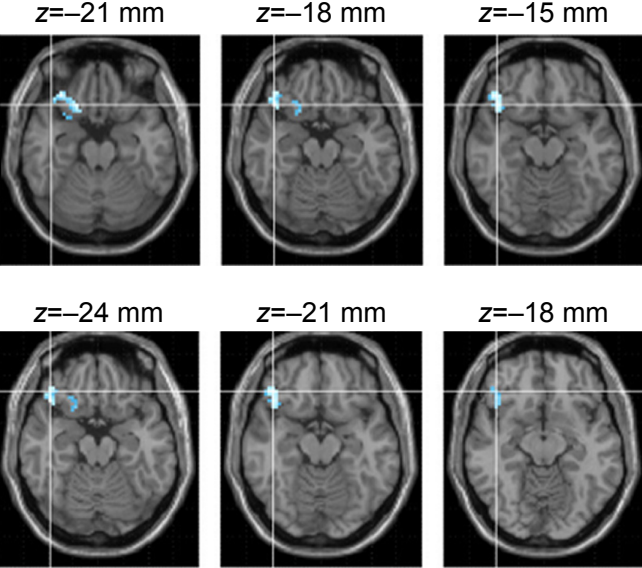

T-value

$t$-value

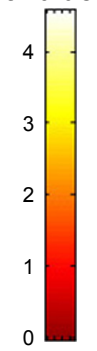

$z=-21 \mathrm{~mm}$

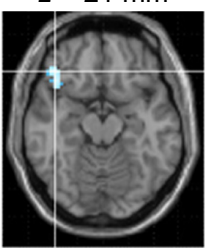

$z=-18 \mathrm{~mm}$
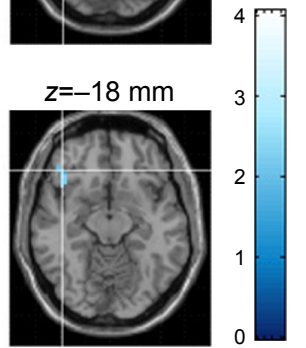

$\mathbf{F}$
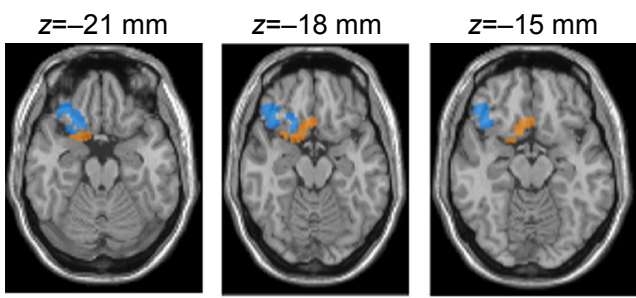

Figure I Affective network in depressive adolescents with disruptive behaviors.

Notes: (A) Lower functional connectivity from the amygdala to the subcallosal area in healthy control participants compared to depressive adolescents with disruptive behaviors (yellow). (B) Lower functional connectivity from the amygdala to the parahippocampal gyrus in healthy control participants compared to depressive adolescents with disruptive behaviors (yellow). (C and D) Amygdala-orbitofrontal cortex connectivity associated with Children Depression Inventory (CDI) score (blue). (E) Correlation between $\mathrm{CDI}$ score and beta value extracted from the amygdala-orbitofrontal cortex. (F) Overlap between figures $\mathbf{A}+\mathbf{B}$ and $\mathbf{C}+\mathbf{D}$.

of the amygdala might also be triggered by the stimulation of negative emotional memories. ${ }^{35}$

\section{RSFC from the PCC to the left precentral gyrus, insula, and parietal lobe} Our current results show higher RSFC of the DMN from the PCC to the left precentral gyrus, left insula, and left parietal lobe in disrup- MDD participants compared to $\mathrm{HC}$ participants. Increased RSFC in the DMN in patients with MDD has been reported in previous RSFC studies. ${ }^{17,36,37}$ Sheline et $\mathrm{al}^{36}$ reported that patients with MDD exhibit increased RSFC from the bilateral dorsomedial prefrontal cortex to the anterior and posterior cingulate cortices and to the precuneus regions. Zhou et $\mathrm{al}^{37}$ reported that patients with

Table 2 Limbic network resting-state connectivity

\begin{tabular}{|c|c|c|c|c|c|c|c|}
\hline \multirow[t]{2}{*}{ Regions } & \multirow[t]{2}{*}{ BA } & \multirow[t]{2}{*}{$\mathbf{k}^{*}$} & \multicolumn{3}{|c|}{ Talairach coordinates } & \multirow[t]{2}{*}{$\mathbf{T} * *$} & \multirow[t]{2}{*}{$\boldsymbol{P}_{\text {uncorrected }}{ }^{* * * *}$} \\
\hline & & & $\bar{x}$ & $y$ & $\mathbf{z}$ & & \\
\hline \multicolumn{8}{|l|}{ Amygdala seed connectivity } \\
\hline \multicolumn{8}{|l|}{$\left({ }_{\text {disrup- }} M D D<H C\right)$} \\
\hline Right subcallosal gyrus & 47 & 99 & 15 & 15 & -12 & 4.38 & $<0.001$ \\
\hline Right parahippocampal gyrus & 34 & 41 & 21 & 6 & -18 & 4.56 & $<0.001$ \\
\hline \multicolumn{8}{|c|}{ CDI scores associated with amygdala } \\
\hline \multicolumn{8}{|c|}{ RSFC in ${ }_{\text {disrup- }}$ MDD patients } \\
\hline Right inferior frontal gyrus & 47 & 101 & 45 & 33 & -18 & 4.04 & $<0.001$ \\
\hline Right inferior frontal gyrus & & & 35 & 33 & -21 & & \\
\hline
\end{tabular}

Notes: $*$, cluster size in voxels. ${ }^{* *} \mathrm{~T}, t$-test statistical value. ${ }^{* * *} P_{\text {uncorrected }}$, uncorrected voxel-wise probability value. $P<0.00 \mathrm{I}$, statistically significant.

Abbreviations: WM, white matter; BA, Brodmann's area; disrup- MDD, patients with major depression and disruptive behavior; HC, healthy controls; CDI, Kovacs' Children Depression Inventory; RSFC, resting-state functional connectivity. 

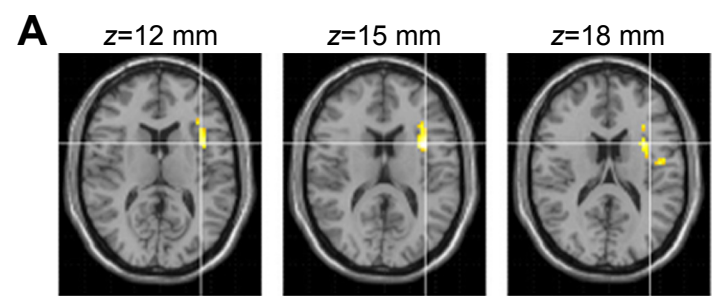

B
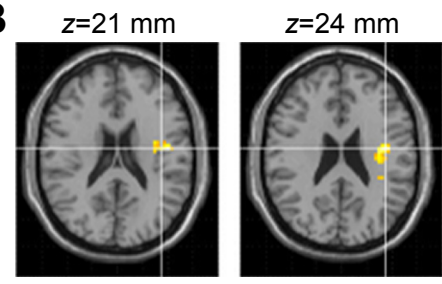

C
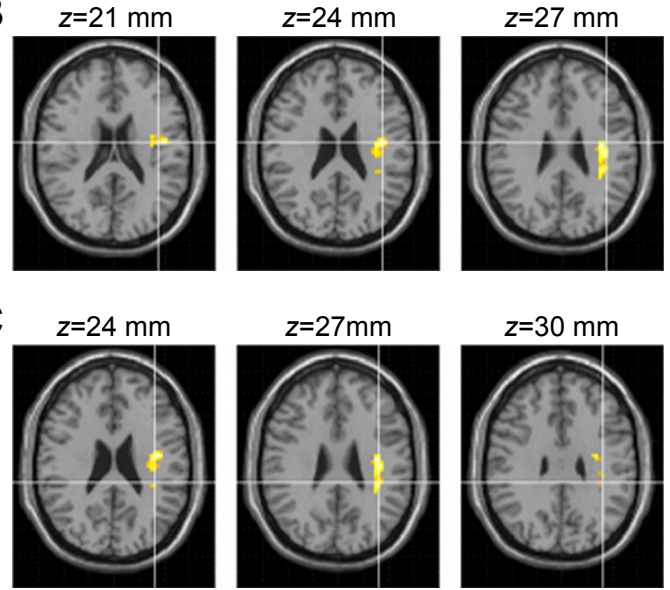

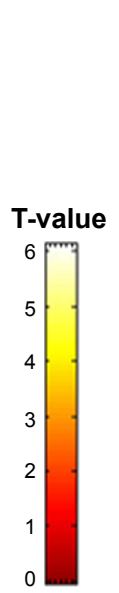

E
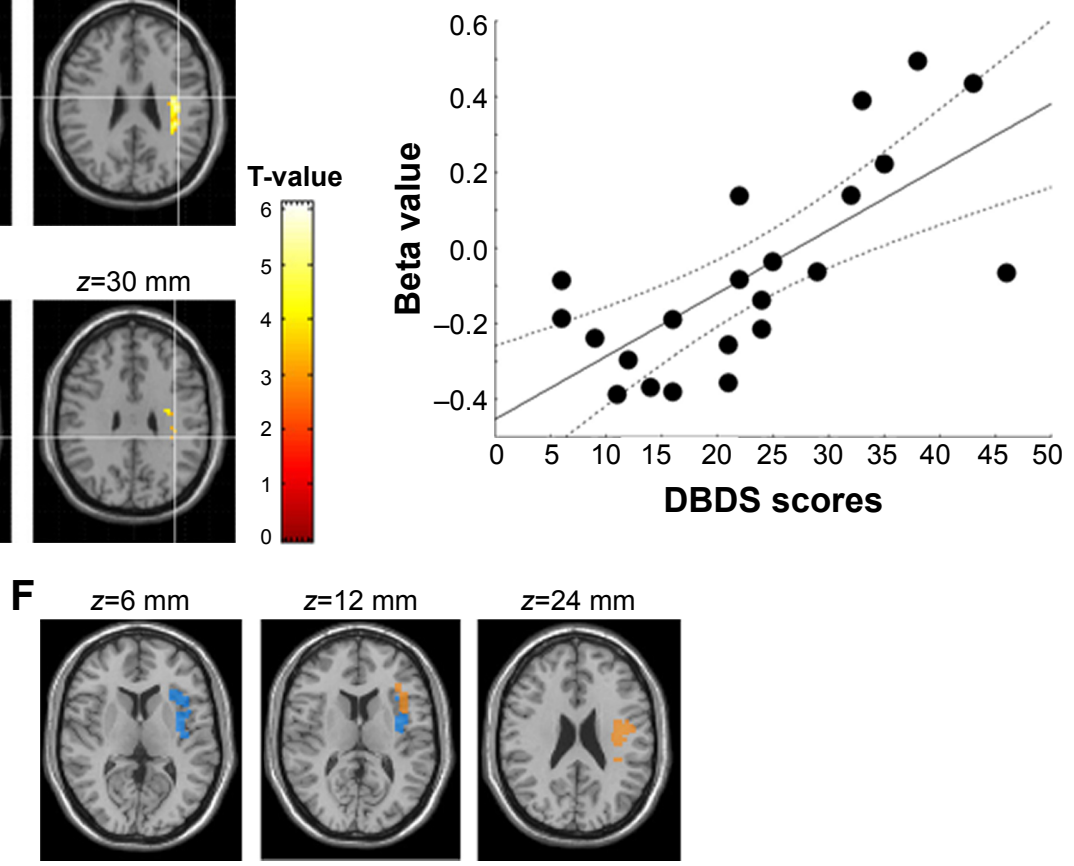

Figure 2 Default mode network in depressive adolescents with disruptive behaviors.

Notes: (A) Higher functional connectivity from the posterior cingulate cortex (PCC) to the insula in healthy control participants compared to depressive adolescents with disruptive behaviors (yellow). (B) Higher functional connectivity from the PCC to the left precentral gyrus in healthy control participants compared to depressive adolescents with disruptive behaviors (yellow). (C) Higher functional connectivity from the PCC to the left inferior parietal lobe in healthy control participants compared to depressive adolescents with disruptive behaviors (yellow). (D) PCC-insular cortex connectivity associated with Rating Scale for Disruptive Behavior Disorder (DBDS) scores (blue). (E) Correlation between DBDS score and beta value extracted from the amygdala-orbitofrontal cortex. (F) Overlap between figures $\mathbf{A}+\mathbf{B}+\mathbf{C}$ and $\mathbf{D}$.

MDD show higher RSFC from the prefrontal cortex to the inferior parietal lobe compared to HCs. Hyperactivity of the DMN is associated with rumination in patients with MDD. ${ }^{38}$ Rumination and negative emotion affect and magnify each other in impulsive people and lead to impulsive behaviors in patients with MDD. ${ }^{39}$ Barrocas et $\mathrm{a}^{40}$ reported that female sex with higher rumination and severity of depression might predict non-suicidal self-injury, based on a study of 617

Table 3 Default mode network resting-state connectivity

\begin{tabular}{|c|c|c|c|c|c|c|c|}
\hline \multirow[t]{2}{*}{ Regions } & \multirow[t]{2}{*}{ BA } & \multirow[t]{2}{*}{$\mathbf{k}^{*}$} & \multicolumn{3}{|c|}{ Talairach coordinates } & \multirow[t]{2}{*}{$\mathbf{T}^{* *}$} & \multirow[t]{2}{*}{$\boldsymbol{P}_{\text {uncorrected }} * * *$} \\
\hline & & & $x$ & $y$ & $\mathbf{z}$ & & \\
\hline \multicolumn{8}{|c|}{ Default mode network - PCC seed } \\
\hline \multicolumn{8}{|c|}{ connectivity $\left(_{\text {disrup. }} M D D<\mathrm{HC}\right)$} \\
\hline Left insula & 13 & 124 & -35 & 9 & 15 & 5.58 & $<0.001$ \\
\hline Left precentral gyrus & WM & 95 & -39 & -9 & 24 & 4.33 & $<0.001$ \\
\hline Left inferior parietal lobe & WM & 121 & -35 & -33 & 27 & 5.01 & $<0.001$ \\
\hline \multicolumn{8}{|c|}{ DBDS scores associated with } \\
\hline \multicolumn{8}{|c|}{ PCC RSFC in disrup $M D D$ patients } \\
\hline Left insula & 13 & 91 & -33 & 12 & 18 & 4.06 & $<0.001$ \\
\hline
\end{tabular}

Notes: ${ }^{*}$, cluster size in voxels. ${ }^{*} \mathrm{~T}$, $t$-test statistical value. ${ }^{* * *} P_{\text {uncorrected }}$, uncorrected voxel-wise probability value. $P<0.00 \mathrm{I}$, statistically significant.

Abbreviations: WM, white matter; BA, Brodmann's area; disrup. MDD, patients with major depression and disruptive behavior; HC, healthy controls; DBDS, Rating Scale for Disruptive Behavior Disorder; PCC, posterior cingulate cortex; RSFC, resting-state functional connectivity. 
Chinese students. Moreover, in the present study, the score

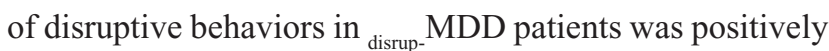
correlated with RSFC from the PCC to the left insular cortex. The insula is thought to have a crucial role in filtering information to promote behavior choice with early evaluation of sensory and affective stimuli, as well as in perceiving the body's physiologic response. ${ }^{41,42}$ Meanwhile, PCC-insula connections are part of both the salience network (cingulate and orbital fronto-insular cortices) and the DMN. ${ }^{43}$ We cautiously speculate that the DMN might be partially connected with the salience network in disrup- MDD patients. Salience network refers to the brain system that plays an important role in identification of relevant stimuli to promote flexible behaviors. ${ }^{44}$ It has been suggested that the salience network controls the down-regulation of the DMN and the upregulation of attention networks. ${ }^{45}$ To date, few studies have investigated the salience network in disruptive behaviors. However, it has been suggested that the key neurobiology of ADHD, which is frequently accompanied by disruptive behaviors, is dysfunction in the salience network that controls the balance between the DMN and attention networks. ${ }^{44,45}$

\section{Limitations}

There were several limitations in the current study. First, the small number of participants might not be sufficient to generalize the results to a larger population and controlling multiple comparisons during analyzing brain data. In addition, we used ROIs of the bilateral amygdala and PCC, while prior work has used ipsilateral and amygdala subregions to show a dissociable functional network. ${ }^{29}$ Finally, the clinical consistency of disrup- MDD patients was not fully confirmed because, in the literature, $12.5 \%-30 \%$ of patients diagnosed with MDD also report later progression to bipolar disorder. ${ }^{46}$ Future studies should include a larger number of participants with a longer observation period and vary across amygdala regions.

\section{Conclusion}

Adolescents with MDD and disruptive behaviors showed lower RSFC from the amygdala to the OFC and the parahippocampal gyrus and higher PCC RSFC compared with HC participants in a cluster that included the left precentral gyrus, left insula, and left parietal lobe. Depressive mood might be correlated with the affective network, and disruptive behavior might be correlated with the DMN.

\section{Acknowledgments}

This study was funded by a grant from the Korean Health Technology R\&D Project, Ministry of Health \& Welfare,
Republic of Korea (A120013); a grant from Student Mental Health Research Center Project through Korea Brain Research Institute supported by the Ministry of Science, ICT \& Future Planning (2231-415, KBRI-20130007); and a grant from the Korea Creative Content Agency (R2014040055).

\section{Disclosure}

The authors report no conflicts of interest in this work.

\section{References}

1. Thapar A, Collishaw S, Pine DS, Thapar AK. Depression in adolescence. Lancet. 2012;379(9820):1056-1067.

2. Copeland WE, Shanahan L, Costello EJ, Angoid A. Childhood and adolescent psychiatric disorders as predictors of young adult disorders. Arch Gen Psychiatry. 2009;66(7):764-772.

3. Stringaris A, Zavos H, Leibenluft E, Maughan B, Eley T. Adolescent irritability: phenotypic associations and genetic links with depressed mood. Am J Psychiatry. 2012;169(1):47-54.

4. Roy A, Oldehinkel AJ, Verhulst FC, Ormel J, Hartman CA. Anxiety and disruptive behavior mediate pathways from attention-deficit/ hyperactivity disorder to depression. J Clin Psychiatry. 2014;75(2): e108-e113.

5. Ezpeleta L, Domenech JM, Angold A. A comparison of pure and comorbid CD/ODD and depression. J Child Psychol Psychiatry. 2006; 47(7):704-712.

6. Simic M, Fombonne E. Depressive conduct disorder: symptom patterns and correlates in referred children and adolescents. J Affect Disord. 2001;62(3):175-185.

7. Bebko G, Bertocci MA, Fournier JC, et al. Parsing dimensional vs diagnostic category-related patterns of reward circuitry function in behaviorally and emotionally dysregulated youth in the Longitudinal Assessment of Manic Symptoms study. JAMA Psychiatry. 2014;71(1):71-80.

8. Raichle ME, MacLeod AM, Snyder AZ, Powers WJ, Gusnard DA, Shulman GL. A default mode of brain function. Proc Natl Acad Sci US A. 2001;98(2):676-682.

9. Foland LC, Altshuler LL, Bookheimer SY, Eisenberger N, Townsend J, Thompson PM. Evidence for deficient modulation of amygdala response by prefrontal cortex in bipolar mania. Psychiatry Res. 2008;162(1):27-37.

10. Ladouceur CD, Farchione T, Diwadkar V, et al. Differential patterns of abnormal activity and connectivity in the amygdala-prefrontal circuitry in bipolar-I and bipolar-NOS youth. J Am Acad Child Adolesc Psychiatry. 2011;50(12):1275-1289, e1272.

11. Bebko G, Bertocci $M$, Chase $H$, et al. Decreased amygdala-insula resting state connectivity in behaviorally and emotionally dysregulated youth. Psychiatry Res. 2015;231(1):77-86.

12. Zeng LL, Shen H, Liu L, et al. Identifying major depression using wholebrain functional connectivity: a multivariate pattern analysis. Brain. 2012; 135(Pt 5):1498-1507.

13. Luking KR, Repovs G, Belden AC, et al. Functional connectivity of the amygdala in early-childhood-onset depression. J Am Acad Child Adolesc Psychiatry. 2011;50(10):1027-1041, e1023.

14. Pannekoek JN, van der Werff SJ, Meens PH, et al. Aberrant resting-state functional connectivity in limbic and salience networks in treatment-naive clinically depressed adolescents. J Child Psychol Psychiatry. 2014; 55(12):1317-1327.

15. Rubia K. "Cool" inferior frontostriatal dysfunction in attention-deficit/ hyperactivity disorder versus "hot" ventromedial orbitofrontal-limbic dysfunction in conduct disorder: a review. Biol Psychiatry. 2011; 69(12): e69-e87.

16. Bluhm RL, Osuch EA, Lanius RA, et al. Default mode network connectivity: effects of age, sex, and analytic approach. Neuroreport. 2008; 19(8):887-891. 
17. Nejad AB, Fossati P, Lemogne C. Self-referential processing, rumination, and cortical midline structures in major depression. Front Hum Neurosci. 2013;7:666.

18. Dalwani MS, Tregellas JR, Andrews-Hanna JR, et al. Default mode network activity in male adolescents with conduct and substance use disorder. Drug Alcohol Depend. 2014;134:242-250.

19. Kim YS, Cheon KA, Kim BN, et al. The reliability and validity of Kiddie-Schedule for Affective Disorders and Schizophrenia-Present and Lifetime Version- Korean version (K-SADS-PL-K). Yonsei Med J. 2004;45(1):81-89.

20. Kovacs M. The Children's Depression Inventory (CDI) Manual. New York: MultiHealth System; 1992.

21. Silva RR, Alpert M, Pouget E, et al. A rating scale for disruptive behavior disorders, based on the DSM-IV item pool. Psychiatr Q. 2005; 76(4):327-339.

22. So YK, Noh JS, Kim YS, Ko SG, Koh YJ. The reliability and validity of Korean parent and teacher ADHD Rating Scale. J Korean Neuropsychiatr Assoc. 2002;41:283-289.

23. Beck AT, Epstein N, Brown G, Steer RA. An inventory for measuring clinical anxiety: psychometric properties. J Consult Clin Psychol. 1988;56(6):893-897.

24. Wechsler D. The Wechsler Intelligence Scale for Children-III. San Antonio, TX: Psychological Corporation; 1991.

25. Maldjian JA, Laurienti PJ, Kraft RA, Burdette JH. An automated method for neuroanatomic and cytoarchitectonic atlas-based interrogation of fMRI data sets. Neuroimage. 2003;19(3):1233-1239.

26. Tzourio-Mazoyer N, Landeau B, Papathanassiou D, et al. Automated anatomical labeling of activations in SPM using a macroscopic anatomical parcellation of the MNI MRI single-subject brain. Neuroimage. 2002;15(1):273-289.

27. Johnstone T, van Reekum CM, Urry HL, Kalin NH, Davidson RJ. Failure to regulate: counterproductive recruitment of top-down prefrontal-subcortical circuitry in major depression. J Neurosci. 2007;27(33): 8877-8884.

28. Rosenkranz JA, Moore H, Grace AA. The prefrontal cortex regulates lateral amygdala neuronal plasticity and responses to previously conditioned stimuli. J Neurosci. 2003;23(35):11054-11064.

29. Cullen KR, Westlund MK, Klimes-Dougan B, et al. Abnormal amygdala resting-state functional connectivity in adolescent depression. JAMA Psychiatry. 2014;71(10):1138-1147.

30. Urry HL, van Reekum CM, Johnstone T, et al. Amygdala and ventromedial prefrontal cortex are inversely coupled during regulation of negative affect and predict the diurnal pattern of cortisol secretion among older adults. J Neurosci. 2006;26(16):4415-4425.

31. Stefanacci L, Suzuki WA, Amaral DG. Organization of connections between the amygdaloid complex and the perirhinal and parahippocampal cortices in macaque monkeys. J Comp Neurol. 1996;375(4): $552-582$.
32. Roy AK, Shehzad Z, Margulies DS, et al. Functional connectivity of the human amygdala using resting state fMRI. Neuroimage. 2009; 45(2):614-626.

33. Smith AP, Stephan KE, Rugg MD, Dolan RJ. Task and content modulate amygdala-hippocampal connectivity in emotional retrieval. Neuron. 2006;49(4):631-638.

34. Versace A, Thompson WK, Zhou D, et al. Abnormal left and right amygdala-orbitofrontal cortical functional connectivity to emotional faces: state versus trait vulnerability markers of depression in bipolar disorder. Biol Psychiatry. 2010;67(5):422-431.

35. Sheline YI, Barch DM, Donnelly JM, et al. Increased amygdala response to masked emotional faces in depressed subjects resolves with antidepressant treatment: an fMRI study. Biol Psychiatry. 2001;50(9): 651-658.

36. Sheline YI, Price JL, Yan Z, Mintun MA. Resting-state functional MRI in depression unmasks increased connectivity between networks via the dorsal nexus. Proc Natl Acad Sci U S A. 2010;107(24):11020-11025.

37. Zhou Y, Yu C, Zheng $\mathrm{H}$, et al. Increased neural resources recruitment in the intrinsic organization in major depression. J Affect Disord. 2010; 121(3):220-230.

38. BroydSJ,DemanueleC,DebenerS,Helps SK, James CJ, Sonuga-BarkeEJS, Default-mode brain dysfunction in mental disorders: a systematic review. Neurosci Biobehav Rev. 2009;33(3):279-296.

39. Selby EA, Kranzler A, Panza E, Fehling KB. Bidirectional-compounding effects of rumination and negative emotion in predicting impulsive behavior: implications for emotional cascades. J Pers. 2014. doi:10.1111/jopy.12147.

40. Barrocas AL, Giletta M, Hankin BL, et al. Nonsuicidal self-injury in adolescence: longitudinal course, trajectories, and intrapersonal predictors. J Abnorm Child Psychol. 2015;43(2):369-380.

41. Craig AD. How do you feel? Interoception: the sense of the physiological condition of the body. Nat Rev Neurosci. 2002;3(8):655-666.

42. Seeley WW, Menon V, Schatzberg AF, et al. Dissociable intrinsic connectivity networks for salience processing and executive control. J Neurosci. 2007;27(9):2349-2356.

43. Elton A, Gao W. Divergent task-dependent functional connectivity of executive control and salience networks. Cortex. 2014;51:56-66.

44. Menon V. Large-scale brain networks and psychopathology: a unifying triple network model. Trends Cogn Sci. 2011;15(10):483-506.

45. Sidlauskaite J, Sonuga-Barke E, Roeyers H, Wiersema JR. Altered intrinsic organisation of brain networks implicated in attentional processes in adult attention-deficit/hyperactivity disorder: a resting-state study of attention, default mode and salience network connectivity. Eur Arch Psychiatry Clin Neurosci. 2015.

46. Gan Z, Diao F, Wei Q, et al. A predictive model for diagnosing bipolar disorder based on the clinical characteristics of major depressive episodes in Chinese population. J Affect Disord. 2011;134(1-3):119-125.
Neuropsychiatric Disease and Treatment

\section{Publish your work in this journal}

Neuropsychiatric Disease and Treatment is an international, peerreviewed journal of clinical therapeutics and pharmacology focusing on concise rapid reporting of clinical or pre-clinical studies on a range of neuropsychiatric and neurological disorders. This journal is indexed on PubMed Central, the 'PsycINFO' database and CAS,
Dovepress

and is the official journal of The International Neuropsychiatric Association (INA). The manuscript management system is completely online and includes a very quick and fair peer-review system, which is all easy to use. Visit http://www.dovepress.com/testimonials.php to read real quotes from published authors. 